\section{RELAÇÃO ENTRE ATIVIDADE FÍSICA, APTIDÃO FÍSICA E RISCO CARDIOVASCULAR: ESTUDO EM MUZAMBINHO, MINAS GERAIS}

\author{
RELATIONSHIP BETWEEN PHYSICAL ACTIVITY, PHYSICAL FITNESS AND CARDIOVASCULARRISK: \\ STUDYINMUZAMBINHO, MINAS GERAIS \\ RELACIÓN ENTRE ACTIVIDAD FÍSICA, APTITUD FÍSICA Y RIESGO CARDIOVASCULAR: ESTUDIO EN \\ MUZAMBINHO, MINAS GERAIS
}

\begin{abstract}
João Paulo dos Anjos
Souza Barbosa

(Profissional de Educação Física)

Luciano Basso ${ }^{2}$

(Profissional de Educação Física)

Teresa Bartholomeu

(Profissional de Educação Física)

António Prista ${ }^{3}$

(Profissional de Educação Física)

Januária Andrea Souza Rezende 4 (Profissional de Educação Física)

Jorge Alberto Oliveira²

(Profissional de Educação Física)

Go Tani²

(Profissional de Educação Física)

José António Ribeiro Maias

(Profissional de Educação Física)

Cláudia Lúcia de Moraes Forjaz’

(Profissional de Educação Física)
\end{abstract}

1. Universidade de São Paulo,

Escola de Educação Física e Esporte,

Laboratório de Hemodinâmica da

Atividade Motora, São Paulo, Brasil.

2. Universidade de São Paulo,

Escola de Educação Física e Esporte,

Laboratório de Comportamento

Motor, São Paulo, Brasil.

3. Universidade Pedagógica, Núcleo

de Investigação em Actividade

Física e Saúde CIDAF-FEFD, Maputo,

Moçambique.

4. Instituto Federal do Sul de

Minas, Campus Muzambinho,

Muzambinho.

5. Universidade do Porto, Faculdade

de Desporto, CIFI²D - Centro

de Investigação, Formação,

Intervenção e Inovação em

Desporto, Laboratório de

Cineantropometria e Gabinete de

Estatística Aplicada, Porto, Portugal.

\section{Correspondência:}

Cláudia Lúcia de Moraes Forjaz

Av. Prof. Mello Moraes, 65, Butantã,

São Paulo, SP, Brasil. 05508-030.

cforjaz@usp.br

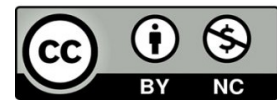

\section{RESUMO}

Introdução: A relação negativa entre atividade física, aptidão física e risco cardiovascular está bem estabelecida em populações com baixos níveis de atividade física. Objetivo: O presente estudo investigou essa relação em adultos de Muzambinho, Minas Gerais, uma população com alta taxa de indivíduos fisicamente ativos. Métodos: Participaram 237 indivíduos (132 mulheres), na maioria jovens (44,3\% entre 20 e 39 anos). Foram avaliados indicadores de risco cardiovascular (índice de massa corporal, circunferência da cintura, glicemia de jejum, colesterolemia de jejum e pressão arterial sistólica e diastólica), atividade física (volume semanal de atividade física total) e aptidão física (aptidão aeróbica e força manual relativa). Resultados: Mais de 90\% dos indivíduos foram classificados como ativos (praticavam atividade física por mais de $150 \mathrm{~min} / \mathrm{semana}$ ). A regressão linear identificou associação negativa da força manual relativa e da aptidão aeróbica ao índice de massa corporal e a circunferência da cintura, bem como associação positiva da força manual relativa à pressão arterial diastólica. Os indicadores de atividade física e aptidão física não se associaram ao risco cardiovascular global. Conclusão: Nessa população, a relação inversa entre atividade física, aptidão física e risco cardiovascular limitou-se aos indicadores de aptidão física e aos de obesidade, sugerindo que em populações com alta taxa de indivíduos fisicamente ativos, o aumento da aptidão física, mas não da atividade física, pode ajudar a reduzir a obesidade. Nível de Evidência ll; ECRC de menor qualidade.

Descritores: Fatores de risco; Atividade motora; Aptidão física; Força muscular.

\section{ABSTRACT}

Introduction: The negative relationship between physical activity, physical fitness, and cardiovascular risk is well established in populations with low levels of physical activity. Objective: The present study investigated this relationship in adults from Muzambinho, Minas Gerais, a population with a high rate of physically active individuals. Methods: Two hundred and thirty-seven individuals (132 women), mostly young adults (44.3\% between 20 and 39 years) were assessed. Cardiovascular risk predictors (body mass index, waist circumference, fasting blood glucose, fasting cholesterol, and systolic and diastolic blood pressure), physical activity (total weekly volume of total physical activity) and physical fitness (aerobic fitness and relative hand strength) were measured. Results: More than $90 \%$ of the individuals were classified as active (they practiced physical activity for more than $150 \mathrm{~min} /$ week). Linear regression identified a negative association of relative hand strength and aerobic fitness to body mass index and waist circumference, as well as a positive association of relative hand strength with diastolic blood pressure. The indicators of physical activity and physical fitness showed no association with global cardiovascular risk. Conclusion: In this population, the inverse relationship between physical activity, physical fitness and cardiovascular risk was limited to physical fitness and obesity indicators, suggesting that in populations with high rates of physically active individuals, the increase in physical fitness, but not in physical activity, can help reduce obesity. Level of Evidence ll; Lesser quality RCT.

Keywords: Risk factors; Motor activity; Physical Fitness; Muscle strength.

\section{RESUMEN}

Introducción: La relación negativa entre actividad física, aptitud física y riesgo cardiovascular está bien establecida en poblaciones con bajos niveles de actividad física. Objetivo: Este estudio investigó esta relación en adultos de Muzambinho, Minas Gerais, una población con una alta tasa de individuos físicamente activos. Métodos: Participaron 237 individuos (132 mujeres), en su mayoría jóvenes (44,3\% entre los 20 y 39 años). Se evaluaron indicadores de riesgo cardiovascular (indice de mas a corporal, circunferencia de la cintura, glucosa en ayunas, colesterol en ayunas y presión arterial sistólica y diastólica), actividad física (volumen semanal de actividad física total) y aptitud física (capacidad aeróbica y fuerza relativa de la mano). Resultados: Más del 90\% de los individuos fueron clasificados como activos (practicaban actividad física por más de $150 \mathrm{~min} / \mathrm{semana}$ ). La regresión lineal identificó asociación negativa de la fuerza relativa de la mano y la capacidad aeróbica al indice de masa corporal y la circunferencia de la cintura, así como la asociación positiva de la fuerza relativa de la mano a la presión arterial diastólica. Los indicadores de actividad física y aptitud física no se asociaron al riesgo cardiovascular global. Conclusión: En esta población, la relación inversa 
entre actividad fisica, aptitud física y riesgo cardiovascular se limitó a los indicadores de aptitud física y obesidad, lo que sugiere que en poblaciones con alta tasa de individuos físicamente activos, el aumento de la aptitud física, pero no de la actividad física, puede ayudar a reducir la obesidad. Nivel de Evidencia ll; ECRC de menor calidad.

Descriptores: Factores de riesgo; Actividad motora; Aptitud física, Fuerza muscular.

\section{INTRODUÇÃO}

A principal causa de morte em adultos no mundo 1 e no Brasil é a doença cardiovascular. Diferentes fatores de risco cardiovascular (FRC) predispõem ao aparecimento dessas doenças, dentre os quais destacam-se para este estudo: a obesidade, o diabetes, a hipercolesterolemia e a hipertensão arterial. Adicionalmente, a inatividade física é também um importante FRC1 1 , sendo considerada a quarta causa de mortalidade no mundo ${ }^{3}$

O controle dos FRC é importante para a redução da morbimortalidade. Porém, as prevalências dos FRC são elevadas no Brasil e no mundo. ${ }^{1,4}$ Segundo o Ministério da Saúde Brasileiro, ${ }^{4}$ a prevalência de excesso de peso em adultos é 48,1\%, diabetes - 7,6\%, hipertensão arterial - 37,8\%, hipercolesterolemia - 20\% e inatividade física - 15\%. ${ }^{5}$ Porém, essas prevalências variam consideravelmente nas diferentes cidades brasileiras e, de maneira geral, foram investigadas em capitais e cidades de médio e grande porte, ${ }^{1,6,7}$ de modo que as altas prevalências são atribuídas às modificações do estilo de vida advindas do desenvolvimento urbano. ${ }^{1}$ Entretanto, a maioria das cidades brasileiras ainda tem pequeno porte e baixo nível de desenvolvimento, podendo apresentar diferentes prevalências dos FRC.

Em 2008, realizamos um estudo em Muzambinho-MG ${ }^{8}$ (cidade de pequeno porte localizada na região Cafeeira do Sul de MG) que possuía na época do estudo 20.430 habitantes (14000 adultos). ${ }^{9}$ Nesse estudo ${ }^{8}$ observamos que, da mesma forma que em cidades de médio e grande porte, as prevalências dos FRC foram consideráveis, principalmente de obesidade (excesso de peso - 44,6\%, glicemia alterada - 1,9\%, pressão arterial alta -16,0\% e colesterol total elevado - 7,4\%). No entanto, contrariamente aos outros estudos, a prevalência de inatividade física foi muito pequena (apenas 4,8\% de insuficientemente ativo ou inativo) e 95,2\% dos adultos foram considerados fisicamente ativos.

Como diversos estudos relatam relação negativa entre a prática de atividade física (AF) e o risco cardiovascular em adultos, ${ }^{10}$ a considerável prevalência FRC observada em Muzambinho em concomitância à alta frequência de AF, contrapõe essa expectativa. Porém, os estudos que relataram a relação negativa foram realizados, em sua maioria, em populações com elevada frequência de pessoas fisicamente inativas, sendo importante avaliar se essa relação permanece em populações com maior frequência de indivíduos fisicamente ativos.

Para contribuir para um melhor entendimento dessas relações em populações com elevada frequência de indivíduos fisicamente ativos, esse estudo investigou a relação entre alguns FRC, considerados de forma isolada e em conjunto, com os níveis de AF e de aptidão física (ApF) nos adultos de Muzambinho - MG.

\section{MATERIAL E MÉTODOS}

Participaram adultos de Muzambinho, que eram pais de crianças que participaram de uma pesquisa sobre os FRC na infância. ${ }^{11}$ Foram avaliados 237 adultos (132 mulheres e 105 homens) de 19 a 65 anos. A prevalência dos FRC em parte dessa amostra já foi publicada. ${ }^{8} \mathrm{O}$ estudo foi aprovado pelo Comitê de Ética em Pesquisa da Escola de Educação Física e Esporte da USP (CAAE-0033.0.342.000-07). Os dados foram coletados de Março/2008 a Agosto/2009.
Os voluntários foram visitados em suas residências para a apresentação do estudo e a assinatura do termo de consentimento. Posteriormente, foi agendada uma segunda visita para a medida de glicemia e colesterolemia de jejum. Em outro dia, os voluntários foram à escola de seus filhos para realizar medidas antropométricas e da pressão arterial (PA), e foi avaliada a prática de AF e a ApF. Após as coletas, foram informados dos resultados das avaliações, receberam orientações de saúde e, quando necessário (FRC presentes e descontrolados), foram encaminhados para acompanhamento médico.

\section{Indicadores de risco cardiovascular}

A massa corporal e a estatura foram medidas (balança Filizola, Brasil). O índice de massa corporal (IMC) foi calculado. A circunferência da cintura (CC) foi medida com uma fita métrica não elástica posicionada na cicatriz umbilical.

A glicemia e colesterolemia foram medidas por monitores automáticos (Accu-Check Advantage, II, Roche, Brasil e Accutrend GC, Roche, EUA, respectivamente) com os voluntários em jejum de, pelo menos, seis horas.

A PA foi medida no braço não dominante após cinco minutos de repouso sentado. As medidas foram realizadas por um avaliador experiente, usando o método auscultatório e um esfigmomanômetro aneroide devidamente calibrado, além de manguitos adequados à circunferência do braço do indivíduo. As fases I e V dos sons de Korotkoff foram utilizadas para determinar, respectivamente, as pressões arteriais sistólica e diastólica. Foram realizadas duas medidas com um intervalo de 30s e, se houvesse uma diferença superior a $4 \mathrm{mmHg}$, uma nova medida era realizada. O valor da PA de cada indivíduo foi estabelecido pela média das duas ou três medidas realizadas. ${ }^{12}$

\section{Indicador de risco cardiovascular global}

Conforme a literatura, ${ }^{13}$ o risco cardiovascular global (RCG) foi calculado para cada indivíduo. O valor de cada FRC medido (IMC, CC, glicemia, colesterolemia, pressão arterial sistólica - PAS e pressão arterial diastólica - PAD) em cada indivíduo foi convertido ao escore $z$, considerando-se o sexo. Em seguida, foi calculado, para cada indivíduo, o somatório de todos os valores z. Assim, quanto menor o somatório, menor o indicador de RCG.

\section{Indicador de AF}

A AF foi avaliada por uma entrevista estruturada ${ }^{8,11}$ realizada por um técnico treinado: os participantes foram questionados sobre as práticas de AF de deslocamento, ocupacional e lazer. Para cada atividade, a frequência semanal e duração foram registradas e o volume semanal foi calculado pela multiplicação da frequência pela duração. O volume semanal de AF total foi calculado pela soma dos volumes relatados de AF de deslocamento, ocupacional e lazer. Foram classificados como fisicamente ativos, os indivíduos que realizavam mais de $150 \mathrm{~min} / \mathrm{se}$ mana de AF total. ${ }^{12}$

\section{Indicadores de ApF}

A aptidão aeróbica foi avaliada pelo teste submáximo de Astrand em cicloergômetro (Monark). ${ }^{14}$ Resumidamente, conforme o sexo e grau de condicionamento físico, foi escolhida uma carga e os indivíduos 
pedalaram por seis min em 50 rpm. A frequência cardíaca foi medida no final do quinto e sexto minutos de exercício. Quando essa frequência estava estável (variação < 4 bpm entre o $5^{\circ}$ e 60. min) e entre 120 e 160 bpm, o teste era finalizado e os dados analisados. Quando a frequência estava abaixo de 120 bpm, a carga era aumentada e se repetia o procedimento. Quando a frequência não se estabilizava ou estava acima de 160 bpm, o teste era finalizado, mas não era analisado devido ao erro na escolha da carga. Os dados coletados corretamente foram analisados no nomograma de Astrand ${ }^{14}$ para se obter uma estimativa de consumo máximo de oxigênio absoluto $\left(\mathrm{VO}_{2} \mathrm{max}, \mathrm{l} / \mathrm{min}\right)$ bruto, que foi corrigido pelo fator de correção de idade ${ }^{15}$ e, posteriormente, dividido pela massa corporal para o cálculo do $\mathrm{VO}_{2}$ max relativo $\left(\mathrm{ml} \cdot \mathrm{kg}^{-1} \cdot \mathrm{min}^{-1}\right)$. Usando a classificação proposta por Astrand, ${ }^{16}$ os indivíduos foram classificados como acima ou abaixo da média prevista para o sexo e a idade.

A força máxima manual foi avaliada pelo teste de preensão manual isométrica no "handgrip" (Saehan Corporation, Hydraulic Hand Dynamometer SH 5001). Após o ajuste da empunhadura, cada voluntário realizou um movimento de preensão manual, utilizando a máxima força possível com o braço dominante posicionado ao lado do corpo. Essa variável foi dividida pela massa corporal, resultando na força manual relativa. Os indivíduos foram classificados como acima ou abaixo da média prevista para o sexo e a idade. ${ }^{17}$

\section{Análise estatística}

Análise exploratória de dados foi feita no SPSS 20 e as frequências, médias e desvios-padrão foram calculados. A normalidade da distribuição dos dados foi verificada pelo teste de Kolmogorov-Smirnov; as variáveis IMC, PAS, colesterolemia, volume semanal de AF total e força manual relativa não tiveram distribuição normal. O teste t ou o teste de Mann-Whitney foram utilizados na comparação entre os sexos. Análises múltiplas de regressão linear robusta foram utilizadas para verificar as associações entre as variáveis dependentes (indicadores de risco cardiovascular isolados e global) e as variáveis independentes (idade, sexo, IMC, volume semanal de AF total, $\mathrm{VO}_{2}$ max relativo e força muscular relativa), utilizando o STATA 13 adotando um $p \leq 0,05$.

\section{RESULTADOS}

A descrição das variáveis está apresentada na Tabela 1. Comparados às mulheres, os homens eram significantemente mais velhos e apresentavam maior CC, PAS, glicemia, colesterolemia e RCG; enquanto que os valores médios do IMC e da PAD não diferiram entre os sexos. O volume semanal de AF total também não diferiu entre os sexos, mas os homens apresentaram valores de $\mathrm{VO}_{2}$ max e força manual relativa significantemente maiores que as mulheres.

Considerando-se o volume de AF total, 94,9\% dos indivíduos (94,4\% dos homens e $95,2 \%$ das mulheres) foram classificados como fisicamente ativos. Além disso, 24,9\% (17,7\% dos homens e 30,9\% das mulheres) tinham aptidão aeróbica acima da média e 63,8\% (65,9\% dos homens e 62,3\% das mulheres) tinham força relativa acima da média.

As análises múltiplas de regressão linear robusta estão na Tabela 2. No modelo múltiplo: i) o IMC se associou negativamente ao sexo feminino, à idade, ao $\mathrm{VO}_{2}$ max e à força manual relativa $\left(r^{2}=0,413\right)$; ii) a CC se associou negativamente ao sexo feminino, à idade, $\mathrm{a} \mathrm{VO}_{2}$ max e à força manual relativa $\left(r^{2}=0,438\right)$; iii) a glicemia se associou negativamente ao sexo feminino $\left(r^{2}=0,156\right)$; iv) a colesterolemia se associou positivamente à idade $\left.\left(r^{2}=0,216\right) ; v\right)$ a PAS se associou positivamente à idade e ao IMC $\left(r^{2}=0,114\right)$; e vi) a PAD se associou positivamente à idade, ao IMC e à força manual relativa $\left(r^{2}=0,240\right)$.

Para o indicador de RCG, o modelo múltiplo revelou associações negativas com o sexo feminino e positivas com a idade e o IMC $\left(r^{2}=0,631\right)$.

\section{DISCUSSÃO}

Os principais resultados desse estudo mostraram associação negativa da aptidão aeróbica e da força manual relativa com o IMC e a CC, bem como associação positiva da força manual relativa com a PAD. O RCG não se associou a nenhum dos indicadores de atividade nem de ApF. Dessa forma, as associações negativas foram evidenciadas apenas entre os indicadores de ApF e os de obesidade.

Considerando-se a AF, diversos estudos sugerem que os seus maiores níveis se associam a menores valores de PA, obesidade, glicemia e colesterolemia em adultos, ${ }^{18-21}$ se relacionando, portanto, a um menor RCG. ${ }^{22}$ Neste estudo, nenhuma relação significante foi evidenciada entre essas variáveis. Essa ausência de associação, pode estar relacionada à frequência de AF elevada da população estudada, tendo em vista que cerca de $95 \%$ dos adultos foram considerados fisicamente ativos, ${ }^{8,11}$ ou seja, faziam mais de 150 minutos/semana de AF. ${ }^{12}$ De fato, vários deles faziam mais de 10 vezes esse volume, uma vez que o alto volume refletia, principalmente, a AF ocupacional e a maioria dos adultos relatou fazer mais de seis horas/diárias de trabalho físico na "roça" ou em atividades domésticas. Assim, os resultados poderiam ser diferentes se a elevada prática de AF se devesse às atividades físicas de lazer, visto que tem sido relatada maior importância dessas atividades na redução do risco cardiovascular. ${ }^{23}$ Assim, os resultados sugerem que em populações

Tabela 1. Valores descritivos de idade e dos indicadores de risco cardiovascular, aptidão física e atividade física medidos na amostra geral e separada por sexo.

\begin{tabular}{|c|c|c|c|c|c|c|c|}
\hline Variáveis & & Geral & & Homens & & Mulheres & \\
\hline & $n$ & Média $\pm \mathrm{DP}$ & $\mathrm{n}$ & Média $\pm \mathrm{DP}$ & $\mathrm{n}$ & Média $\pm \mathrm{DP}$ & $p$ \\
\hline Idade (anos) & 237 & $36 \pm 7$ & 105 & $38 \pm 8$ & 132 & $35 \pm 6$ & 0,001 \\
\hline \multicolumn{8}{|l|}{ Indicadores de risco cardiovascular } \\
\hline IMC $\left(\mathrm{kg} \cdot \mathrm{m}^{-2}\right)$ & 212 & $25,7 \pm 4,1$ & 90 & $25,6 \pm 3,3$ & 122 & $25,7 \pm 4,6$ & 0,790 \\
\hline $\mathrm{CC}(\mathrm{cm})$ & 212 & $88 \pm 10$ & 90 & $90 \pm 9$ & 122 & $87 \pm 11$ & 0,050 \\
\hline $\mathrm{GLI}(\mathrm{mg} / \mathrm{dL})$ & 227 & $91 \pm 11$ & 99 & $95 \pm 12$ & 128 & $89 \pm 10$ & 0,000 \\
\hline PAD $(\mathrm{mmHg})$ & 211 & $76 \pm 12$ & 90 & $78 \pm 12$ & 121 & $75 \pm 12$ & 0,132 \\
\hline RCG (Escore $\boldsymbol{z})$ & 234 & $0,00 \pm 3,54$ & 105 & $0,83 \pm 3,39$ & 129 & $-0,68 \pm 3,52$ & 0,001 \\
\hline \multicolumn{8}{|l|}{ Indicadores de aptidão física } \\
\hline $\mathrm{VO}_{2} \max \left(\mathrm{ml}^{\prime} \mathrm{kg}^{-1} \cdot \mathrm{min}^{-1}\right)$ & 173 & $31 \pm 7$ & 79 & $32 \pm 8$ & 94 & $30 \pm 7$ & 0,042 \\
\hline Força manual relativa ( $\mathrm{kg} / \mathrm{kg}$ de peso) & 208 & $0,51 \pm 0,12$ & 88 & $0,59 \pm 0,10$ & 120 & $0,46 \pm 0,11$ & 0,000 \\
\hline
\end{tabular}

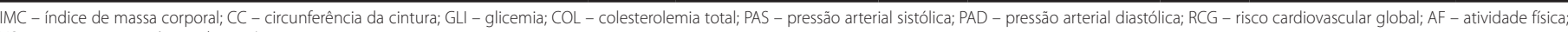
$\mathrm{VO}_{2}$ max - consumo máximo de oxigênio. 
Tabela 2. Estimativas ajustadas dos modelos de regressão, relativos à associação do sexo, idade, aptidão física e atividade física com os indicadores de risco cardiovascular isolados e risco global.

\begin{tabular}{|c|c|c|c|}
\hline & $\mathrm{R}^{2}$ & $\beta$ & IC 95\% \\
\hline IMC & 0,413 & & \\
\hline Sexo & & $-2,766^{* * *}$ & $(-3,873 ;-1,658)$ \\
\hline Idade (anos) & & $-0,079 *$ & $(-0,153 ;-0,004)$ \\
\hline $\mathrm{VO}_{2} \max \left(\mathrm{ml} \cdot \mathrm{kg}^{-1} \cdot \mathrm{min}^{-1}\right)$ & & $-0,293^{* * *}$ & $(-0,362 ;-0,224)$ \\
\hline Força manual relativa & & $-8,914^{* * *}$ & $(-13,325 ;-4,503)$ \\
\hline AF Total (min/sem) & & 0,000 & $(-0,000 ; 0,000)$ \\
\hline $\mathrm{CC}$ & 0,438 & & \\
\hline Sexo & & $-9,772^{* * *}$ & $(-12,715 ;-6,828)$ \\
\hline Idade (anos) & & $-0,220^{*}$ & $(-0,417 ;-0,022)$ \\
\hline $\mathrm{VO}_{2} \max \left(\mathrm{ml} \cdot \mathrm{kg}^{-1} \cdot \mathrm{min}^{-1}\right)$ & & $-0,791^{* * *}$ & $(-0,974 ;-0,608)$ \\
\hline Força manual relativa & & $-23,502^{* * *}$ & $(-35,225 ;-11,779)$ \\
\hline AF Total (min/sem) & & 0,000 & $(-0,000 ; 0,002)$ \\
\hline GLI & 0,156 & & \\
\hline Sexo & & $-6,959 * *$ & $(-11,019 ;-2,900)$ \\
\hline Idade (anos) & & 0,088 & $(-0,171 ; 0,348)$ \\
\hline $\mathrm{IMC}\left(\mathrm{kg} \cdot \mathrm{m}^{-2}\right)$ & & 0,430 & $(-0,069 ; 0,929)$ \\
\hline $\mathrm{VO}_{2} \max \left(\mathrm{ml} \cdot \mathrm{kg}^{-1} \cdot \mathrm{min}^{-1}\right)$ & & $-0,004$ & $(-0,299 ; 0,290)$ \\
\hline Força manual relativa & & $-7,791$ & $(-23,932 ; 8,350)$ \\
\hline AF Total (min/sem) & & $-0,000$ & $(-0,002 ; 0,000)$ \\
\hline $\mathrm{COL}$ & 0,216 & & \\
\hline Sexo & & $-8,900$ & $(-21,937 ; 4,136)$ \\
\hline Idade (anos) & & $0,892 *$ & $(0,127 ; 1,656)$ \\
\hline $\mathrm{IMC}\left(\mathrm{kg} \cdot \mathrm{m}^{-2}\right)$ & & 1,042 & $(-0,436 ; 2,522)$ \\
\hline $\mathrm{VO}_{2} \max \left(\mathrm{ml} \cdot \mathrm{kg}^{-1} \cdot \mathrm{min}^{-1}\right)$ & & $-0,619$ & $(-1,524 ; 0,285)$ \\
\hline Força manual relativa & & 10,423 & $(-42,426 ; 63,272)$ \\
\hline AF Total (min/sem) & & $-0,003$ & $(-0,008 ; 0,001)$ \\
\hline PAS & 0,114 & & \\
\hline Sexo & & $-2,258$ & $(-7,170 ; 2,654)$ \\
\hline Idade (anos) & & $0,372 *$ & $(0,056 ; 0,687)$ \\
\hline$I M C\left(k g \cdot \mathrm{m}^{-2}\right)$ & & $0,635 *$ & $(0,034 ; 1,235)$ \\
\hline $\mathrm{VO}_{2} \max \left(\mathrm{ml} \cdot \mathrm{kg}^{-1} \cdot \mathrm{min}^{-1}\right)$ & & $-0,021$ & $(-0,368 ; 0,325)$ \\
\hline Força manual relativa & & 12,188 & $(-7,368 ; 31,745)$ \\
\hline AF Total (min/sem) & & $-0,000$ & $(-0,002 ; 0,001)$ \\
\hline PAD & 0,240 & & \\
\hline Sexo & & 1,747 & $(-2,012 ; 5,507)$ \\
\hline Idade (anos) & & $0,552^{* * *}$ & $(0,311 ; 0,793)$ \\
\hline $\mathrm{IMC}\left(\mathrm{kg} \cdot \mathrm{m}^{-2}\right)$ & & $0,945^{* * *}$ & $(0,458 ; 1,404)$ \\
\hline $\mathrm{VO}_{2} \max \left(\mathrm{ml} \cdot \mathrm{kg}^{-1} \cdot \mathrm{min}^{-1}\right)$ & & 0,086 & $(-0,178 ; 0,352)$ \\
\hline Força manual relativa & & $19,762 * *$ & $(4,794 ; 34,731)$ \\
\hline AF Total (min/sem) & & $-0,001$ & $(-0,003 ; 0,000)$ \\
\hline RCG & 0,631 & & \\
\hline Sexo & & $-1,461 * * *$ & $(-2,357 ;-0,566)$ \\
\hline Idade (anos) & & 0,097 *** & $(0,040 ; 0,155)$ \\
\hline$I M C\left(k g \cdot \mathrm{m}^{-2}\right)$ & & 0,606 *** & $(0,496 ; 0,716)$ \\
\hline $\mathrm{VO}_{2} \max \left(\mathrm{ml} \cdot \mathrm{kg}^{-1} \cdot \mathrm{min}^{-1}\right)$ & & $-0,014$ & $(-0,078 ; 0,048)$ \\
\hline Força manual relativa & & 0,826 & $(-2,750 ; 4,404)$ \\
\hline AF Total (min/sem) & & $-0,000$ & $(-0,000 ; 0,000)$ \\
\hline
\end{tabular}

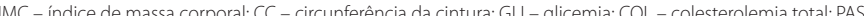
pressão arterial sistólica; PAD - pressão arterial diastólica; RCG - risco cardiovascular global; AF - atividade física; pressão arterial sistólica; PAD - pressão arterial diastólica; RCG - risco cardiova
$\mathrm{VO}_{2} \mathrm{max}$ - consumo máximo de oxigênio; ${ }^{*} p<0,05 ;{ }^{* *} p<0,01 ;{ }^{* * *} p<0,001$

fisicamente ativas devido à elevada prática de AF no trabalho, a relação negativa entre o nível de AF total e o risco cardiovascular não é evidente.

Quanto aos indicadores de ApF, a aptidão aeróbica e a força muscular relativa se associaram negativamente ao IMC e a CC, mas não se relacionaram com a glicemia, a colesterolemia, a PA e o RCG. Na literatura, estudos demonstraram que, tanto em homens quanto em mulheres, melhores níveis de aptidão cardiorrespiratória e de força muscular associam-se a menores valores dos FRC ${ }^{24}$ e também com o RCG. ${ }^{18-21}$. Assim, os resultados deste estudo corroboram apenas em parte os da literatura, restringindo a associação negativa apenas aos indicadores de obesidade. A ausência de efeito nos demais indicadores pode estar associada às características da população estudada, principalmente, ao fato de se caracterizar como uma população de adultos jovens (cerca de 50\% entre 20 e 39 anos) e, portanto, com baixa prevalência de FRC alterados, fato este diferente do que apresenta a literatura. ${ }^{23}$

Apesar da ausência de associação com os indicadores destacados acima, o resultado com maior impacto do presente estudo, foi a presença de associação negativa entre os indicadores de ApF e os de obesidade geral e central. Dessa forma, os adultos com maior potência aeróbica e maior força muscular relativa dos membros superiores apresentavam menor IMC e menor CC. Ressalta-se que a população estudada apresentou elevada prevalência de excesso de peso $(53,8 \%)$ e CC alterada (29,7\%), apesar dos altos níveis de AF. Dessa forma, os resultados indicam que mesmo numa população adulta jovem e ativa, o excesso de peso pode ser um problema e o aumento da ApF, mas não do nível de AF, pode ter impacto positivo sobre os indicadores de obesidade, sugerindo que intervenções nesse tipo de população devam visar a melhora da aptidão aeróbica e da força e não apenas aumentar ainda mais o nível de AF. De fato, já tem sido discutida a maior importância da melhora da ApF sobre o nível de AF para a redução do risco cardiovascular, ${ }^{25}$ o que parece ser ainda mais importante em populações com elevados níveis de AF total, como a população estudada. Nesse contexto, intervenções de atividades físicas estruturadas são mais eficazes para o desenvolvimento de componentes específicos de $\mathrm{ApF}^{26}$ e, portanto, talvez esse tipo de intervenção deva ser utilizado em populações com as características da população estudada nessa investigação.

Estranhamente, no presente estudo, em vez de apresentar associação negativa, a PAD se associou positivamente com a força manual relativa, ou seja, os indivíduos mais fortes apresentavam maior PAD. A razão para essa relação não está clara. Poder-se-ia pensar que o resultado estivesse vinculado ao tamanho da circunferência do braço, ou seja, indivíduos com mais força teriam braços maiores e o que poderia levar a um erro de medida, superestimando o valor da PA nos indivíduos com maior circunferência de braço. ${ }^{27}$ No entanto, o uso de manguitos adequados à circunferência do braço, como feito nesse estudo, elimina essa explicação. Assim, essa relação positiva ainda carece de melhor explicação.

Os FRC medidos nos adultos apresentaram associação com o sexo, a idade e o IMC. O sexo feminino se associou a menor IMC, CC, glicemia e, portanto, ao menor RCG, o que está de acordo com a proposição de que a mulher, principalmente em idade fértil, apresenta menor risco cardiovascular que o homem. ${ }^{28}$ Em relação à idade, houve associação positiva com colesterolemia, PAS, PAD e RCG, demonstrando que indivíduos mais velhos tinham maiores valores de todos esses indicadores, como amplamente conhecido. ${ }^{12}$ Além disso, a idade se associou negativamente com o IMC e com a CC, sugerindo que dentro dessa população jovem, os mais novos têm maiores índices de obesidade geral e central, o que torna o problema da obesidade ainda mais evidente. Considerando-se o IMC, como tem sido extensivamente relatado na literatura, ${ }^{29}$ ele se associou positivamente com os demais FRC analisados de forma isolada e com o RCG.

Não obstante a importância dos achados, o presente estudo apresentou algumas limitações. Devido ao seu desenho transversal, uma relação de causa e efeito não pode ser indicada, nem a direção de qualquer possível influência. Devido à época em que o estudo foi desenvolvido e à ausência do conhecimento prévio do tipo de prática física realizada, o estudo utilizou uma entrevista estruturada, que foi usada anteriormente, ${ }^{11,30}$ para avaliar a AF, e os resultados podem ser diferentes com outros questionários ou com medidas diretas de $A F$, o que precisa ser testado. Dessa forma, é importante ressaltar que os resultados devem ser interpretados à luz da forma como foram avaliados, principalmente na comparação com outros estudos. 
Além disso, a amostra foi estabelecida por conveniência e envolveu pais de crianças do ensino fundamental e, portanto, a maior parte era formada por adultos jovens, de modo que os resultados não podem ser extrapolados para a população adulta em geral.

\section{CONCLUSÃO}

Houve associação negativa da aptidão aeróbica e da força manual relativa com o IMC e a CC, e associação positiva entre a força manual relativa e a PAD. Porém, o indicador de AF não se associou a nenhum indicador de risco cardiovascular isolado e o RCG não se associou a nenhum indicador de AF nem de ApF nos adultos de Muzambinho-MG.

\section{AGRADECIMENTOS}

Os autores agradecem à Secretaria Municipal de Educação e de Saúde de Muzambinho pelo apoio à execução deste estudo; aos professores e dirigentes das instituições de ensino, que participaram deste projeto; aos monitores, que participaram da coleta de dados do projeto; e aos voluntários deste estudo. O estudo teve apoio financeiro do CNPq (478249/2007-1), da Pró-Reitoria de Graduação da USP (2009.1.9397.1.9) e da CAPES.

Todos os autores declararam não haver qualquer potencial conflito de interesses referente a este artigo.

CONTRIBUICÕES DOS AUTORES: Cada autor contribuiu individual e significativamente para o desenvolvimento deste estudo. JPASB (0000-0002-1813-6040)*, AP (0000-0002-3652-7205)*, GT (0000-0002-6517-5472)*, JARM e CLMF participaram da concepção desse artigo. LB (0000-0002-3301-4877)*, TB (0000-0002-37576682)*, AP, JASR (0000-0001-9240-5467)*, JAO (0000-0002-0703-4639)*, CLMF participaram da coleta de dados. JPASB, TB, JASR, AP, LB, JAO, JARM (0000-0002-3273-0046)* e CLMF (0000-0001-7584-4265)* participaram da tabulação e análise dos dados. Todos os autores participaram ativamente da discussão dos resultados. JPASB, JARM e CLMF foram os principais contribuintes na redação do manuscrito. Todos realizaram a revisão do manuscrito. *ORCID (Open Researcher and Contributor ID).

\section{REFERÊNCIAS}

1. Cannon, C.P., Cardiovascular disease and modifiable cardiometabolic risk factors. Clin Cornerstone. 2007;8(3):11-28.

2. Ministério da Saúde. Cadernos de informações de saúde. 2009. [acesso em 2016 jul 6]. Disponível em: http://www.datasus.gov.br.

3. World Health Organization World health statistics 2013: a wealth of information on global public health. Geneva: World Health Organization; 2013.

4. DATASUS. Ministério da Saúde. Secretaria de Gestão Estratégica e Participativa - Departamento de Monitoramento e Avaliação da Gestão do SUS. 2006 Disponível em: http://www.datasus.gov.br.

5. Ministério da Saúde. Vigilância de fatores de risco e proteção para doenças crônicas por inquérito telefônico. 2015 [acesso em 2016 jul 6]; Disponível em: http://portalsaude.saude.gov.br/portalsaude/arquivos/pdf/2012/Mai/09/Vigitel_2011_diabetes_final.pdf.

6. De Ferranti SD, Osganian SK. Epidemiology of pediatric metabolic syndrome and type 2 diabetes mellitus. Diab Vasc Dis Res. 2007;4(4):285-96.

7. Froberg K, Andersen LB. Mini review: physical activity and fitness and its relations to cardiovascular disease risk factors in children. Int J Obes. 2005;29(2):34-9.

8. Barbosa JP,Bartolomeu T, Rezende JA, Basso L, Oliveira JA,Tani G, et al. Risco cardiovascular e prática de atividade física em adultos de Muzambinho/MG: influência do gênero e da idade. Rev Bras Ativ Fís Saúde. 2013;18(2):242-52

9. IBGE, Instituto Brasileiro de Geografia e Estatística. Ministério da Educação, Instituto Nacional de Estudos e Pesquisas Educacionais - INEP - Censo da Educação Superior. 2008

10. ACSM, ACSM's Guidelines for Exercise Testing and Prescription 2010, Philadelphia: Lippincott Williams and Wilkins.

11. Chehuen MdR, Bezerra AlL, BartholomeuT, Junqueira NO, Rezende JAS, Basso L, et al. Risco cardiovascular e prática de atividade física em crianças e adolescentes de Muzambinho/MG: influência do gênero e da idade. Rev Bras Med Esporte. 2011;17:232-6.

12. Sociedade Brasileira de Cardiologia. Sociedade Brasileira de Hipertensão. e Sociedade Brasileira de Nefrologia. VI Diretrizes brasileiras de hipertensão arterial. 2016.

13. Andersen LB, Hasselstrøm H, Grønfeldt V, Froberg K, Hansen SE. The relationship between physical fitness and clustered risk, and tracking of clustered risk from adolescence to young adulthood: eight years follow-up in the Danish Youth and Sport Study. Int J Behav Nutr Phys. 2004;1 (1):6.

14. Astrand PO, Ryhming I. A nomogram for calculation of aerobic capacity (physical fitness) from pulse rate during sub-maximal work. J Appl Physiol. 1954;7(2):218-21.

15. Heyward VH, Gibson A. Advanced fitness assessment and exercise prescription. $7^{\text {th }}$ ed. Champaign: Human Kinetics; 1997.
16. Åstrand PO. Rodahl K, Dahl HA, Stromme SB. Textbook of work physiology: physiological bases of exercise $4^{\text {th }}$ ed. Champaign: Human Kinetics: 2003.

17. Adam Claude, Council of Europe. Committee for the Development of Sport. Council of Europe Committee of Experts on Sports Research. Eurofit, Handbook for the Eurofit tests of physical fitness, in Council of Europe Committee for the development of sport. Roma: Italian Nacional Olympic Committee, Centra Direction for Sport's Technical Activities Documentation an Information Division; 1988

18. Paffenbarger RS,Jr, Wing AL, Hyde RT, Jung DL. Physical activity and incidence of hypertension in college alumni. Am J Epidemiol. 1983;117(3):245-57.

19. Lemmens VE, Oenema A, Klepp KI, Henriksen HB, Brug J. A systematic review of the evidence regarding efficacy of obesity prevention interventions among adults. Obesity Reviews. 2008;9(5):446-55.

20. Jeon CY, Lokken RP, Hu FB, van Dam RM. Physical activity of moderate intensity and risk of type 2 diabetes. Diabetes Care. 2007:30(3):744-52

21. Matsudo VK, Matsudo SM, Araújo TL, Ribeiro MA. Dislipidemias e a promoção da atividade física: uma revisão na perspectiva de mensagens de inclusão. Rev Bras Ci Mov. 2005;13(2):161-70.

22. D'Agostino RB, Vasan RS, Pencina MJ, Wolf PA, Cobain M, Massaro JM, ,et al. General cardiovascular risk profile for use in primary care: the Framingham Heart Study. Circulation. 2008;117(6):743-53.

23. Shiroma EJ, Lee I-M, Physical activity and cardiovascular health: lessons learned from epidemiological studies across age, gender, and race/ethnicity. Circulation. 2010;122(7):743-52.

24. Sassen B, Cornelissen VA, Kiers H, Wittink H, Kok G, Vanhees L. Physical fitness matters more than physica activity in controlling cardiovascular disease risk factors. Eur J Cardiovasc Prev Rehabil. 2009;16(6):677-83.

25. Blair SN, Cheng Y, Holder JS. Is physical activity or physical fitness more important in defining health benefits? Med Sci Sports Exerc. 2001;33(6 SUPP): S379-S399.

26. Garber CE, Blissmer B, Deschenes MR, Franklin BA, Lamonte MJ, Lee IM. American College of Sports Medicine position stand. Quantity and quality of exercise for developing and maintaining cardiorespiratory, musculoskeletal, and neuromotor fitness in apparently healthy adults: guidance for prescribing exercise Med Sci Sports Exerc, 2011;43(7):1334-59.

27. Veiga EV, Arcuri EA, Cloutier L, Santos JL. Medida da pressão arterial: circunferência braquial e disponibilidade de manguitos. Rev Latino-Am Enfermagem. 2009;17(4):455-61

28. Luz PL, Solimene MC. Peculiaridades da doença arterial coronária na mulher. Rev Assoc Med Bras. 1999:45:45-54

29. Francischi RP, Pereira LO, Freitas CS, Klopfer M, Santos RC Vieira P et al. Obesidade: atualização sobre sua etiologia, morbidade e tratamento. Rev Nutr. 2000;13(1):17-28.

30. Forjaz CL, Bartolomeu T, Rezende JA, Oliveira JA, Basso L, Tani G, et al. Genetic and environmenta influences on blood pressure and physical activity: a study of nuclear families from Muzambinho, Brazil. Braz J Med Biol Res. 2012;45(12):1269-75. 